\title{
Differential diagnosis of Marfan syndrome based on ocular biologic parameters
}

\author{
Yiyao Wang, Zhangkai Lian, Yijing Zhou, Xuepei Li, Jieyi Wu, Xinyu Zhang, Guangming Jin, \\ Danying Zheng
}

State Key Laboratory of Ophthalmology, Zhongshan Ophthalmic Center, Sun Yat-sen University, Guangzhou, China

Contributions: (I) Conception and design: D Zheng, G Jin; (II) Administrative support: D Zheng, G Jin; (III) Provision of study materials or patients: D Zheng; (IV) Collection and assembly of data: Y Wang, Z Lian, Y Zhou, X Li, J Wu; (V) Data analysis and interpretation: Y Wang, G Jin; (VI) Manuscript writing: All authors; (VII) Final approval of manuscript: All authors.

Correspondence to: Prof. Danying Zheng, MD; Guangming Jin, MD, PhD. State Key Laboratory of Ophthalmology, Zhongshan Ophthalmic Center, Sun Yat-sen University, No. 54 Xianlie South Road, Yuexiu District, Guangzhou, China. Email: zhengdyy@163.com; jingm@mail2.sysu.edu.cn.

Background: The present study aimed to investigate the ocular characteristics of Chinese patients with Marfan syndrome (MFS) and to evaluate the sensitivity and specificity of ocular parameters in distinguishing MFS from ectopia lentis.

Methods: A total of 103 patients (103 eyes) with ectopia lentis from 13 provinces and 47 cities were recruited from Zhongshan Ophthalmic Center, Sun Yat-sen University, from June 2017 to June 2019. Ghent-2 criteria were used, as they are the gold standard diagnostic criteria for MFS. Ocular parameters, such as flat keratometry (Kf), steep keratometry (Ks), mean keratometry $(\mathrm{Km})$, corneal astigmatism (AST), axial length (AL), white-to-white corneal diameter (WTW), central corneal thickness (CCT), and axial length/curvature radius (AL/CR), were recorded. Diagnostic analyses based on various combinations of parameters to differentiate MFS from ectopia lentis were made using receiver-operating characteristic (ROC) curves.

Results: The mean age of the 103 included patients was $10.25 \pm 9.67$ (range: $3-48$ ) years, and $66.02 \%$ were male. Km combined with CCT was found to have good sensitivity and specificity in the differential diagnosis of MFS from ectopia lentis; Km of $41.36 \mathrm{D}$ and CCT of $537.32 \mathrm{~mm}$ were found to be the optimal cut-off points, representing a sensitivity of $89.8 \%$ and specificity of $68.7 \%$.

Conclusions: Special characteristics of ocular parameters were detected in MFS. Our findings indicate that ocular biologic parameters are valuable for the differential diagnosis of MFS from ectopia lentis. Km, combined with CCT, could be used as a screening tool for MFS.

Keywords: Marfan syndrome (MFS); ocular biologic parameters; diagnosis

Submitted Jan 30, 2020. Accepted for publication Sep 18, 2020.

doi: 10.21037/atm-20-1069

View this article at: http://dx.doi.org/10.21037/atm-20-1069

\section{Introduction}

Marfan syndrome (MFS) is a severe, autosomal-dominant inherited disease with a prevalence of $1 / 5,000-1 / 10,000$ $(1,2)$. The leading cause of MFS is thought to be a fibrillin-1 (FBN1) mutation, which usually causes pathological changes in FBN1 in connective tissues and induces an inherited disorder involving several systems, including the cardiovascular and skeletal systems $(3,4)$. The ocular manifestations can be lens subluxation, or they may be associated with other ocular abnormalities, including high myopia, secondary glaucoma, and retinal detachment. MFS is also accompanied by skeletal system disorders, including arachnodactyly, pectus carinatum, and scoliosis (5). Lesions involving connective tissue, particularly in the cardiovascular system, causing mitral 
valve insufficiency, artery dilation, and aortic aneurysms, can be fatal $(6,7)$. Early intervention is critical because of the life-threatening complications associated with MFS. A previous study showed that losartan, which is generally used for the treatment of hypertension, has the potential to prevent the significant life-threatening manifestations of MFS (8). Regarding ocular disorders, surgery has become the principal treatment for severe ectopia lentis; auxiliary instruments, such as capsular tension rings, have shown promise in visual rehabilitation (9).

The 2010 Revised Ghent Criteria are the gold standard criteria for the diagnosis of MFS (10); the main diagnostic items are as follows: (I) FBN1 gene test; (II) ectopia lentis; (III) the cardiac score; and (IV) the systemic score. Of these four items, gene testing is the most important criteria. However, according to a survey conducted by the Zhongshan Ophthalmic Center, Chinese patients and their parents have low acceptance of genetic testing (11). Furthermore, most cardiovascular complications appear late and usually do not show significant clinical symptoms in young patients with ectopia lentis. Therefore, it is difficult for ophthalmologists to make a differential diagnosis of MFS from with ectopia lentis according to the 2010 Revised Ghent Criteria.

We have observed that, of all MFS symptoms, lens dislocation is usually the most common and easily observed; therefore, patients often consult ophthalmologists first. Even though much attention has been paid to the clinical manifestations and progression of ectopia lentis $(12,13)$, and several previous studies showing that the ocular parameters of MFS patients differ from those of normal controls and that they have particular characteristics $(14,15)$, it remains unknown as to whether these parameters could be used for the differential diagnosis of MFS from ectopia lentis $(16,17)$. Ocular examinations are much cheaper and more acceptable than those mentioned above. If ocular biologic parameters could be used to differentiate MFS from ectopia lentis, this would benefit both patients and clinicians. Therefore, the present study aimed to determine the potential value of biologic parameters in the differential diagnosis of MFS from ectopia lentis. We present the following article following the TRIPOD reporting checklist (available at http://dx.doi.org/10.21037/atm-20-1069).

\section{Methods}

The present case-control study was conducted at the Zhongshan Ophthalmic Center, Sun Yat-sen University,
Guangzhou, China. The study was conducted following the Declaration of Helsinki (as revised in 2013) and was approved by the Institutional Board of Zhongshan Ophthalmic Centre of Sun Yat-sen University (IRBZOC-SYSU). Informed consent was obtained from all the participants.

\section{Participants}

A total of 103 patients (103 eyes) patients with congenital lens dislocation were recruited from Zhongshan Ophthalmic Center of Sun Yat-sen University during July 2017 and June 2019. Of these, 52 patients were diagnosed with MFS based on the Ghent-2 criteria (Table S1). A total of 51 age- and sex-matched patients with ectopia lentis who did not meet the Ghent diagnostic criteria were selected as non-MFS patients. The right eye of each patient was selected for subsequent analyses.

The inclusion criteria were participants with congenital ectopia lentis. The exclusion criteria were as follows: (I) patients with secondary lens dislocation, such as ocular and head trauma; (II) patients with ocular surgery history that could affect the ocular parameters; and (III) patients with a history of retinal detachment or any other disease that may affect the relevant ocular parameters.

\section{Examinations}

All patients underwent detailed binocular examinations and systemic examinations by experienced clinicians. Biologic parameters of the anterior segment, including flat keratometry (Kf), steep keratometry (Ks), mean keratometry $(\mathrm{Km})$, corneal astigmatism (AST), axial length (AL), whiteto-white corneal diameter (WTW), and axial length/ curvature radius (AL/CR) were collected by IOL Master (Carl Zeiss Meditec, Jena, Germany), central corneal thickness (CCT) data were collected via the Pentacam HR system (Oculus, Wetzlar, Germany). Patients' family and medical histories were evaluated after the examinations.

\section{Genetic testing}

Genomic DNA of the proband and available family members were extracted from peripheral blood using the TIANamp blood DNA kit (Tiangen Biotech, Beijing, China), according to the manufacturer's instructions. DNA quantity and quality were verified using NanoDrop (Thermo Fisher Scientific, Waltham, MA, USA). Based on the 
Table 1 Demographic characteristics of MFS and non-MFS patients

\begin{tabular}{|c|c|c|c|c|}
\hline Characteristics & Total & MFS & Non-MFS & $\mathrm{P}$ \\
\hline Age (years), mean \pm SD & $10.25 \pm 9.67$ & $10.52 \pm 9.50$ & $9.55 \pm 8.18$ & 0.399 \\
\hline Male, $\mathrm{n}(\%)$ & $68(66.02)$ & $37(71.15)$ & $31(60.78)$ & 0.267 \\
\hline Z score & $0.75 \pm 2.26$ & $2.18 \pm 2.29$ & $-0.39 \pm 1.45$ & 0.015 \\
\hline
\end{tabular}

MFS, Marfan syndrome; SD, standard deviation; AO, aortic diameter.

reported spectrum, we captured the sequence of the coding region of the FBN1 gene using the SeqCap EZ Library SR V5 kit from NimbleGen (Roche, Basel, Switzerland). Paired libraries were generated using the KAPA HTP Library Preparation Kit (Roche, Basel, Switzerland), and then sequencing with the Illumina MiSeq v2 kit (300 cycles PE) on the Illumina Miseq platform (Illumina, USA). The average sequencing depth was $>100 \times$. Raw data sequencing (FASTQ format) used the Strand NGS software Run Local Realignment Recalibrate Base Qualities program for sequence comparisons. SNP was used to detect single nucleotide variations (SNP) and insertion deletion variations (INDEL). The Detection SNP Effect Analysis program was used to compare dbNSEP v2b3 for mutation screening and annotation, which were compared with ReFseq Genes database.

\section{Variant analysis}

All FBN1 gene variants of sequencing data were selected and screened using the following steps: (I) variations with a frequency $>0.01$ in the 1,000 Genomes Project and ExAC database were excluded; (II) synonymous variations or noncoding region variations that did not affect splicing were excluded; (III) 9 bioinformatics analysis software platforms (Polyphen2_HVAR, SIFT, LRT_pred, Polyphen2_ HDIV, MutationTaster, MutationAssessor, MetaSVM, MetaLR, and FATHMM) were used to predict amino acid substitutions that would change the structures of proteins and affect their functions; and (IV) GERP++ RS software and PhyloP software were used to analyze the conservation of variations. A variation was considered a candidate pathogenic mutation when the result of damage or probability damage in $>3$ of the 9 software platforms and 2 conservative analysis software platforms found a variationconservative missense variation. Sanger sequencing was performed to confirm the mutation, and SWISS-MODEL software was used to predict the structure of the abnormal protein.

\section{Statistical analysis}

Quantitative data with normal distribution were described as mean \pm standard deviation (SD); $t$-tests were performed for intergroup comparisons. The value of biologic parameters in the differential diagnosis of MFS was analyzed using the receiver-operating characteristic (ROC) curve of patients. The test level was set at $\alpha=0.05 ; \mathrm{P}<0.05$ was statistically significant. All statistical analyses were performed using SPSS version 22.0 (IBM, Armonk, NY, USA).

\section{Results}

\section{Demographic characteristics and ocular parameters of all included patients}

A total of 103 patients with ectopia lentis from 13 provinces and 47 cities were included in the present study. The mean age of the patients was $10.25 \pm 9.67$ (range: $3-48$ ) years, and $66.02 \%$ were male. The demographic characteristics of the included patients are provided in Table 1. To analyze differences in ocular biometric characteristics, we divided patients into four groups by age: G1 (3-6 years), G2 (7-12 years), G3 (13-18 years), and G4 ( $>18$ years).

Differences in ocular parameters between the left and right eye of patients with ectopia lentis are shown in Table 2. There were no significant differences in Kf, Ks, Km, AST, AL, WTW, CCT, or AL/CR in terms of ocular parameters between the left and right eye, suggesting that each eye would be reasonable to use for analysis. Based on these results, we only included the right eye parameters for subsequent analyses. 


\section{Age-specific characteristics and ocular parameters of MFS and non-MFS patients}

The demographic characteristics of MFS and non-MFS patients are shown in Table 3. There were no significant differences in terms of age and sex distribution in each age group between MFS and non-MFS patients. Also, there were no significant differences in terms of visual acuity (VA) or intraocular pressure (IOP) between the MFS and nonMFS groups in each age group.

The basic ocular characteristics of the MFS and nonMFS patients are shown in Table 4. Significant differences between $\mathrm{Kf}$ and Ks were found in the G1-G3 groups. Moreover, there were significant differences in AL, CCT, and Km between MFS and non-MFS patients, and MFS patients had longer AL $(25.16 \pm 2.12$ vs. $24.54 \pm 2.18$, $\mathrm{P}=0.011)$, reduced CCT $(516.29 \pm 31.67$ vs. $542.32 \pm 47.94$, $\mathrm{P}=0.029)$, and lower keratometry $(40.47 \pm 1.71$ vs. $41.49 \pm 1.71$, $\mathrm{P}=0.006)$ compared with non-MFS patients. There were no significant differences in AST $(1.79 \pm 1.13$ vs. $1.92 \pm 1.07 \mathrm{D}$, $\mathrm{P}=0.584)$, WTW (12.25 \pm 0.56 vs. $12.25 \pm 1.16 \mathrm{~mm}, \mathrm{P}=0.982)$, or AL/CR (3.03 \pm 0.29 vs. $3.01 \pm 0.29, \mathrm{P}=0.993)$ between MFS and non-MFS patients.

\section{ROC curves of ocular parameters about the diagnosis of MFS}

To explore the potential value of biologic parameters in the differential diagnosis of MFS, parameters with positive statistical significance between MFS and non-MFS were further analyzed using ROC curves. As shown in Figures 1,2, the ROC curve indicated that $\mathrm{Km}$, CCT, and AL were able to discriminate between MFS and non-MFS. The area under the ROC curve (AUROC) was 0.761 for AL, 0.736 for $\mathrm{Km}, 0.713$ for CCT, 0.825 for the combination of $\mathrm{Km}$ and CCT, 0.807 for the combination of $\mathrm{Km}$ and $\mathrm{AL}$, and 0.816 for the combination of CCT and AL; Km of $41.36 \mathrm{D}$ and CCT of $537.32 \mathrm{~mm}$ were found to be the optimal cutoff points, representing a sensitivity of $89.8 \%$ and specificity of $68.7 \%$.

\section{Discussion}

Previous studies have suggested that MFS is associated with distinctive clinical ocular manifestations, including AL enlargement, corneal flattening, reduced CCT, and ectopia lentis $(18,19)$. Several studies have confirmed these biometric characteristics over the past 30 years $(20,21)$.
In the current study, we investigated the characteristics of ocular parameters in MFS patients and found increased AL and decreased $\mathrm{Km}$ and CCT. Ocular biologic parameters are essential for the differential diagnosis of MFS from ectopia lentis; Km combined with CCT could be used as a screening tool for MFS.

In the present study, we found a significant difference in AL between MFS and non-MFS patients $(\mathrm{P}=0.011)$. The average AL of MFS patients was significantly longer than that of non-MFS, which was consistent with the findings of previous studies. Gehle et al. reported on 285 German patients with MFS with ages ranging from 1 to 75 years old. They found that the AL $(24.25 \pm 1.74 \mathrm{~mm})$ of patients with MFS was significantly longer than that of the control group $(23.89 \pm 1.31 \mathrm{~mm})(16)$. Studies have indicated that MFS patients with lens dislocation have longer AL than those without lens dislocation. A study of 87 Norwegians found that their AL with congenital dislocation was significantly longer $(24.99 \pm 3.27 \mathrm{~mm})$ than patients without congenital dislocation $(24.58 \pm 1.33 \mathrm{~mm})$ (18). Several other studies have found that MFS patients with retinal detachment have a longer AL. In a study of 39 patients with MFS in China, patients with retinal detachment had a significantly longer AL (26.5-29.2 $\mathrm{mm}$ ) than patients without retinal detachment (23.8-27.7 mm) (22). Taken together with the results of the current study, the data suggest that a longer AL appears to be a characteristic of MFS; therefore, more attention should be paid to the AL elongation.

Previous studies have reported that MFS patients have decreased cornea keratometry (23-25). Our results also showed that there was a significant difference in corneal curvature between MFS and the non-MFS patients $(\mathrm{P}=0.006)$. The average Km of MFS patients was $40.47 \pm 1.71 \mathrm{D}$, which was lower than that of non-MFS patients. Sultan et al. investigated 31 patients with MFS and found that the average corneal curvature $(40.8 \pm 1.4 \mathrm{D})$ was significantly smaller than that of normal controls $(42.9 \pm 1.1 \mathrm{D})(26)$. MFS patients with lens dislocation have lower keratometry than those without lens dislocation. Drolsum et al. found that the average corneal curvature of MFS patients with lens dislocation was $41.55 \pm 1.73$ (range: $37.63-45.00$ ) $\mathrm{D}$, and the average corneal curvature of patients without lens dislocation was $42.01 \pm 1.68 \mathrm{D}$ (range: $39.88-46.50 \mathrm{D}, \mathrm{P}<0.05)(18)$. These data suggest that corneal curvature may be a useful screening tool for the diagnosis of MFS.

In the present study, we also showed that CCT in MFS patients was significantly reduced compared with non-MFS patients, which was consistent with the findings of previous 
Table 2 Differences in ocular parameters between the left and right eye of patients with ectopia lentis

\begin{tabular}{|c|c|c|c|c|c|c|c|c|c|c|c|c|c|c|c|}
\hline \multirow{2}{*}{ Parameters } & \multicolumn{3}{|c|}{ Total $(n=206)$} & \multicolumn{3}{|c|}{ 3-6 years $(n=84)$} & \multicolumn{3}{|c|}{$7-12$ years $(n=62)$} & \multicolumn{3}{|c|}{$13-18$ years $(n=16)$} & \multicolumn{3}{|c|}{$>18$ years $(n=44)$} \\
\hline & $\mathrm{OD}(\mathrm{n}=103)$ & OS (n=103) & $P$ & $\mathrm{OD}(\mathrm{n}=42)$ & OS (n=42) & $P$ & $\mathrm{OD}(\mathrm{n}=31)$ & OS $(n=31)$ & $P$ & $O D(n=8)$ & OS $(n=8)$ & $P$ & $\mathrm{OD}(\mathrm{n}=22)$ & OS (n=22) & $\mathrm{P}$ \\
\hline $\mathrm{Kf}(\mathrm{D})$ & $40.11 \pm 1.73$ & $40.00 \pm 1.76$ & 0.666 & $39.50 \pm 1.36$ & $39.17 \pm 1.39$ & 0.304 & $40.25 \pm 1.79$ & $40.18 \pm 1.69$ & 0.884 & $40.53 \pm 1.63$ & $40.55 \pm 1.85$ & 0.985 & $40.98 \pm 1.97$ & $41.25 \pm 1.72$ & 0.649 \\
\hline Ks (D) & $41.94 \pm 1.99$ & $41.93 \pm 1.94$ & 0.967 & $40.99 \pm 1.70$ & $41.02 \pm 1.74$ & 0.929 & $42.13 \pm 1.76$ & $42.04 \pm 1.69$ & 0.850 & $42.67 \pm 1.73$ & $42.77 \pm 1.76$ & 0.937 & $43.30 \pm 1.85$ & $43.34 \pm 1.77$ & 0.941 \\
\hline Km (D) & $41.03 \pm 1.78$ & $40.97 \pm 1.78$ & 0.813 & $40.24 \pm 1.46$ & $40.10 \pm 1.45$ & 0.662 & $41.19 \pm 1.73$ & $41.11 \pm 1.64$ & 0.863 & $41.61 \pm 1.86$ & $41.66 \pm 1.79$ & 0.958 & $42.14 \pm 1.80$ & $42.30 \pm 1.67$ & 0.779 \\
\hline AST (D) & $1.83 \pm 1.06$ & $1.94 \pm 1.04$ & 0.522 & $1.49 \pm 1.00$ & $1.85 \pm 1.22$ & 0.160 & $1.88 \pm 0.78$ & $1.86 \pm 0.84$ & 0.925 & $2.13 \pm 1.01$ & $2.21 \pm 0.47$ & 0.891 & $2.32 \pm 1.25$ & $2.09 \pm 1.02$ & 0.536 \\
\hline $\mathrm{AL}(\mathrm{mm})$ & $24.90 \pm 2.19$ & $24.67 \pm 2.22$ & 0.488 & $24.41 \pm 1.83$ & $24.12 \pm 1.62$ & 0.475 & $24.83 \pm 2.19$ & $24.74 \pm 2.47$ & 0.892 & $25.40 \pm 1.52$ & $25.87 \pm 1.89$ & 0.641 & $25.20 \pm 2.55$ & $25.67 \pm 2.29$ & 0.509 \\
\hline WTW (mm) & $12.13 \pm 0.50$ & $12.17 \pm 0.76$ & 0.776 & $12.27 \pm 0.49$ & $12.25 \pm 0.60$ & 0.837 & $12.20 \pm 0.50$ & $11.95 \pm 0.55$ & 0.105 & $12.05 \pm 0.32$ & $11.85 \pm 0.62$ & 0.505 & $11.75 \pm 0.42$ & $12.13 \pm 0.35$ & 0.251 \\
\hline $\mathrm{CCT}(\mathrm{mm})$ & $537.54 \pm 36.75$ & $540.85 \pm 42.87$ & 0.649 & $530.52 \pm 39.20$ & $533.23 \pm 34.00$ & 0.812 & $538.10 \pm 40.61$ & $540.05 \pm 41.01$ & 0.885 & $527.00 \pm 19.61$ & $531.00 \pm 24.96$ & 0.809 & $548.05 \pm 31.72$ & $553.41 \pm 56.21$ & 0.735 \\
\hline
\end{tabular}

OD, oculus dexter; OS, oculus sinister; Kf, flat keratometry; Ks, steep keratometry; Km, mean keratometry; AST, corneal astigmatism; AL, axial length; WTW, white-to-white corneal diameter; CCT, central corneal thickness; AL/CR, axial length/curvature radius.

Table 3 Basic characteristics of patients with and without a diagnosis of MFS

\begin{tabular}{|c|c|c|c|c|c|c|c|c|c|c|c|c|c|c|c|}
\hline \multirow[b]{2}{*}{ Characteristics } & \multicolumn{3}{|c|}{ Total $(n=103)$} & \multicolumn{3}{|c|}{$3-6$ years $(n=42)$} & \multicolumn{3}{|c|}{$7-12$ years $(n=31)$} & \multicolumn{3}{|c|}{$13-18$ years $(n=8)$} & \multicolumn{3}{|c|}{$>18$ years $(n=22)$} \\
\hline & MFS $(n=52)$ & $\begin{array}{c}\text { Non-MFS } \\
(\mathrm{n}=51)\end{array}$ & $P$ & MFS $(n=26)$ & $\begin{array}{c}\text { Non-MFS } \\
(\mathrm{n}=16)\end{array}$ & P & MFS $(n=17)$ & $\begin{array}{c}\text { Non-MFS } \\
(\mathrm{n}=14)\end{array}$ & P & MFS (n=2) & Non-MFS (n=6) & $\mathrm{P}$ & MFS (n=7) & $\begin{array}{c}\text { Non-MFS } \\
(n=15)\end{array}$ & P \\
\hline Male (\%) & 71.15 & 60.78 & 0.267 & 80.76 & 68.75 & 0.239 & 70.58 & 71.42 & 0.981 & 50.00 & 33.33 & 0.173 & 71.42 & 60.00 & 0.251 \\
\hline Age (year) & $10.52 \pm 9.50$ & $9.55 \pm 8.18$ & 0.399 & $4.80 \pm 0.89$ & $4.75 \pm 0.93$ & 0.843 & $8.22 \pm 1.83$ & $8.14 \pm 1.29$ & 0.721 & $17.00 \pm 1.41$ & $15.80 \pm 1.78$ & 0.261 & $26.85 \pm 9.85$ & $25.93 \pm 8.78$ & 0.299 \\
\hline VA (logMAR) & $1.10 \pm 0.43$ & $1.01 \pm 0.32$ & 0.260 & $1.09 \pm 0.41$ & $0.87 \pm 0.31$ & 0.127 & $1.12 \pm 0.44$ & $1.02 \pm 0.35$ & 0.260 & $1.26 \pm 0.05$ & $1.11 \pm 0.23$ & 0.204 & $1.03 \pm 0.54$ & $1.06 \pm 0.40$ & 0.890 \\
\hline IOP (mmHg) & $13.29 \pm 3.20$ & $14.14 \pm 3.93$ & 0.505 & $13.07 \pm 3.10$ & $13.44 \pm 3.49$ & 0.737 & $12.79 \pm 3.07$ & $13.60 \pm 3.09$ & 0.550 & $16.00 \pm 4.04$ & $15.15 \pm 3.45$ & 0.502 & $14.80 \pm 3.43$ & $15.38 \pm 3.65$ & 0.605 \\
\hline
\end{tabular}

MFS, Marfan syndrome; VA, visual acuity; MAR, minimum angle of resolution; IOP, intraocular pressure.

Table 4 Ocular biologic parameters of patients with and without a diagnosis of MFS

\begin{tabular}{|c|c|c|c|c|c|c|c|c|c|c|c|c|c|c|c|}
\hline \multirow[b]{2}{*}{ Parameters } & \multicolumn{3}{|c|}{ Total $(n=103)$} & \multicolumn{3}{|c|}{$3-6$ years $(n=42)$} & \multicolumn{3}{|c|}{$7-12$ years $(n=31)$} & \multicolumn{3}{|c|}{$13-18$ years $(n=8)$} & \multicolumn{3}{|c|}{$>18$ years $(\mathrm{n}=22)$} \\
\hline & MFS (n=52) & $\begin{array}{l}\text { Non-MFS } \\
(\mathrm{n}=51)\end{array}$ & P & MFS (n=26) & $\begin{array}{l}\text { Non-MFS } \\
(\mathrm{n}=16)\end{array}$ & P & MFS (n=17) & $\begin{array}{c}\text { Non-MFS } \\
(n=14)\end{array}$ & P & MFS $(n=2)$ & Non-MFS (n=6) & P & MFS $(n=7)$ & $\begin{array}{c}\text { Non-MFS } \\
(n=15)\end{array}$ & P \\
\hline $\mathrm{Kf}(\mathrm{D})$ & $39.58 \pm 1.65$ & $40.52 \pm 1.73$ & 0.010 & $39.29 \pm 1.41$ & $39.46 \pm 1.33$ & 0.045 & $39.92 \pm 1.81$ & $40.65 \pm 1.66$ & 0.016 & $40.72 \pm 0.44$ & $41.64 \pm 1.11$ & 0.028 & $40.44 \pm 2.01$ & $40.55 \pm 1.58$ & 0.221 \\
\hline Ks (D) & $41.37 \pm 1.95$ & $42.45 \pm 1.85$ & 0.009 & $40.71 \pm 1.62$ & $41.41 \pm 1.88$ & 0.034 & $41.69 \pm 1.71$ & $42.51 \pm 1.42$ & 0.024 & $41.18 \pm 0.43$ & $42.33 \pm 1.01$ & 0.009 & $42.53 \pm 2.04$ & $42.59 \pm 1.58$ & 0.228 \\
\hline Km (D) & $40.47 \pm 1.71$ & $41.49 \pm 1.71$ & 0.006 & $40.00 \pm 1.43$ & $40.44 \pm 1.46$ & 0.037 & $40.81 \pm 1.71$ & $41.59 \pm 1.50$ & 0.025 & $40.95 \pm 0.20$ & $41.89 \pm 0.88$ & 0.007 & $41.39 \pm 2.09$ & $41.57 \pm 1.50$ & 0.076 \\
\hline AST (D) & $1.79 \pm 1.13$ & $1.92 \pm 1.07$ & 0.584 & $1.41 \pm 1.00$ & $1.95 \pm 1.41$ & 0.184 & $1.77 \pm 0.83$ & $1.85 \pm 0.77$ & 0.805 & $1.46 \pm 1.27$ & $1.68 \pm 1.24$ & 0.514 & $2.09 \pm 1.33$ & $2.03 \pm 0.95$ & 0.959 \\
\hline $\mathrm{AL}(\mathrm{mm})$ & $25.16 \pm 2.12$ & $24.54 \pm 2.18$ & 0.011 & $24.71 \pm 1.93$ & $24.00 \pm 1.54$ & 0.049 & $25.58 \pm 2.21$ & $24.55 \pm 2.36$ & 0.014 & $26.58 \pm 1.77$ & $25.64 \pm 1.75$ & 0.028 & $27.56 \pm 2.27$ & $25.49 \pm 2.22$ & 0.005 \\
\hline WTW (mm) & $12.25 \pm 0.56$ & $12.25 \pm 1.16$ & 0.982 & $12.39 \pm 0.51$ & $12.28 \pm 0.39$ & 0.523 & $12.26 \pm 0.56$ & $12.14 \pm 0.58$ & 0.592 & $11.75 \pm 0.07$ & $12.05 \pm 0.36$ & 0.343 & $11.90 \pm 0.40$ & $12.15 \pm 1.68$ & 0.403 \\
\hline $\mathrm{CCT}(\mathrm{mm})$ & $516.29 \pm 31.67$ & $542.32 \pm 47.94$ & 0.029 & $512.08 \pm 39.00$ & $540.00 \pm 40.20$ & 0.038 & $514.40 \pm 27.60$ & $543.57 \pm 36.28$ & 0.044 & $518.00 \pm 19.61$ & $540.50 \pm 20.56$ & 0.035 & $513.50 \pm 34.59$ & $545.54 \pm 32.82$ & 0.047 \\
\hline $\mathrm{AL} / \mathrm{CR}$ & $3.03 \pm 0.29$ & $3.01 \pm 0.29$ & 0.993 & $2.92 \pm 0.23$ & $2.87 \pm 0.15$ & 0.458 & $3.08 \pm 0.26$ & $2.97 \pm 0.38$ & 0.371 & $3.16 \pm 0.21$ & $3.01 \pm 0.15$ & 0.377 & $3.12 \pm 0.33$ & $3.09 \pm 0.29$ & 0.840 \\
\hline
\end{tabular}

MFS, Marfan syndrome; Kf, flat keratometry; Ks, steep keratometry; Km, mean keratometry; AST, corneal astigmatism; AL, axial length; WTW, white-to-white corneal diameter; CCT, central corneal thickness; ALCR, axial length/curvature radius. 

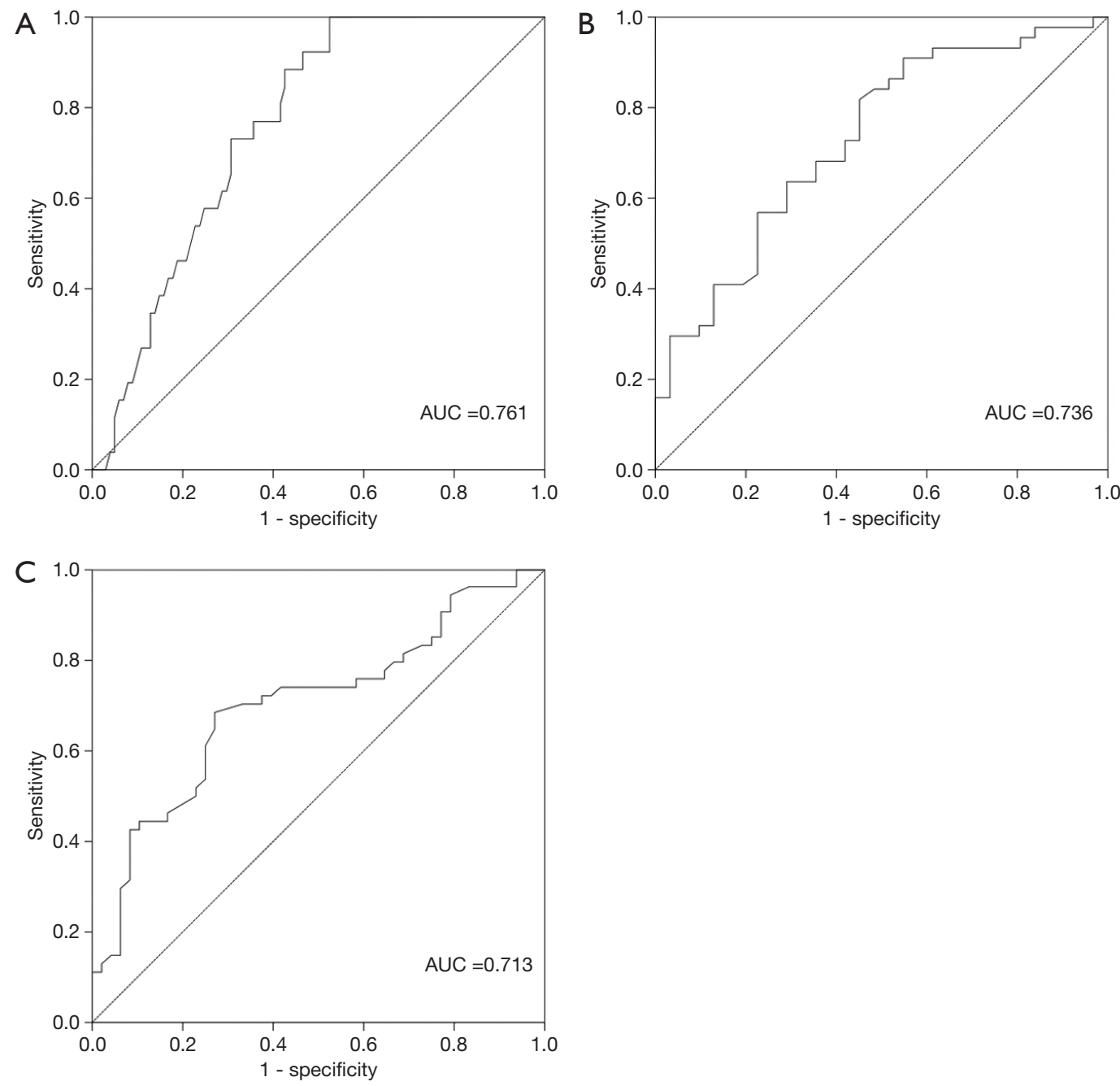

Figure 1 ROC curves of ocular parameters in MFS diagnosis. (A) ROC curves of AL; (B) ROC curves of Km; (C) ROC curves of CCT. ROC, receiver-operating characteristic; MFS, Marfan syndrome; AL, axial length; Km, mean keratometry; CCT, central corneal thickness; AUC, area under the curve.

studies $(\mathrm{P}=0.029)$. In a US study of 62 patients, the CCT of MFS patients was significantly reduced compared to the normal control group (27). Kara et al. found that the CCT of 38 MFS patients was reduced compared to the control group; however, the difference was not statistically significant (24). We believe these inconsistencies may be caused by differences in sample size or ethnic differences. The potential value of CCT in the diagnosis of MFS should also be considered.

A recent study showed that more than half of Chinese patients and their families do not accept genetic testing. The reasons for this include its high cost and lack of evidence regarding its safety (11). Because ocular parameters are different between MFS and non-MFS patients, it is necessary to investigate the potential values of this biologic information when making a differential diagnosis of MFS from ectopia lentis, especially considering that the measurement of biologic parameters is a noninvasive, cheaper, and safer method compared with genetic testing. As seen in Figures 1,2, Km, CCT, and AL had potential diagnostic value for MFS patients, further confirming the different characteristics between MFS and non-MFS patients reported in previous studies $(14,24)$. Our findings suggest that, for patients with congenital lens dislocation, those with $\mathrm{AL}>26.33 \mathrm{~mm}$ and/or $\mathrm{Km}<41.36 \mathrm{D}$, and/or reduced CCT $<537.32 \mathrm{~mm}$, are at high risk of MFS. 

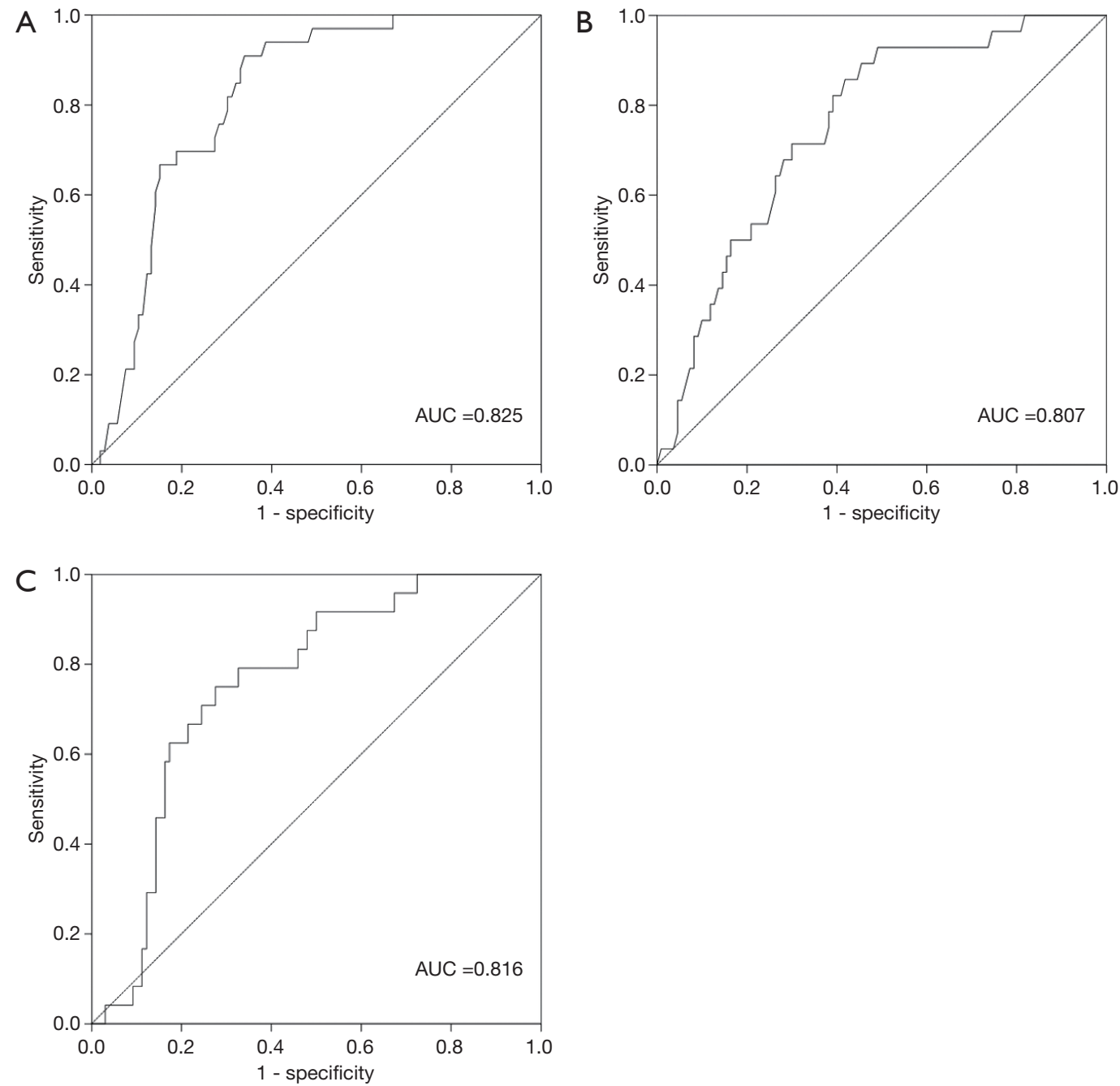

Figure 2 ROC curves of combined ocular parameters in MFS diagnosis. (A) ROC curves for the combination of Km and CCT; (B) ROC curves for the combination of $\mathrm{Km}$ and AL; (C) ROC curves for the combination of CCT and AL. ROC, receiver-operating characteristic; MFS, Marfan syndrome; Km, mean keratometry; CCT, central corneal thickness; AL, axial length; AUC, area under the curve.

When combining $\mathrm{Km}$ and CCT, Km of $41.36 \mathrm{D}$ and CCT of $537.32 \mathrm{~mm}$ were found to be optimal cut-off points (AUROC: 0.825 , sensitivity: $89.8 \%$, specificity: $68.7 \%$ ). These results are consistent with those of a previous study that included 55 Chinese MFS patients. The findings of that study suggested that patients with lens dislocation had $\mathrm{Km}<41.35 \mathrm{D}$, which indicates the diagnostic value in the differential diagnosis of MFS from ectopia lentis (AUROC: 0.85 , sensitivity: $84.9 \%$, specificity: $70.9 \%$ ) (28). Considering the special characteristics and good sensitivity and specificity of the ocular parameters in MFS diagnosis, ocular parameters are valuable in the differential diagnosis of MFS from ectopia lentis and should be given more attention.

Although the current study had a relatively large sample size for a rare disease, ectopia lentis, there were some limitations. First, the sensitivity and specificity of the MFS diagnostic test might not apply to different populations due to racial differences. Second, although the sample size was quite large, and patients came from different provinces of China, the single-center design of the current study limits the generalization of our conclusions to some extent.

We recommend that $\mathrm{Km}$ and CCT, which are acceptable and easily performed for ectopia lentis patients, be considered as the first screening tools in the differential diagnosis of MFS from ectopia lentis. Another benefit 
of these quick and simple screening tools is that genetic testing can be performed in more targeted patients to avoid unnecessary invasiveness and high costs. Nevertheless, this is not intended to diminish the significance of genetic testing, because genetic testing is not only very helpful for the differential diagnosis of MFS from ectopia lentis, but can also be used for investigating other conditions.

In summary, we identified special characteristics of ocular parameters in MFS patients, including longer AL, flatter $\mathrm{Km}$, and reduced CCT. The current study provides new evidence, suggesting that ocular parameters are not only sensitive but also specific, in the differential diagnosis of MFS from ectopia lentis.

\section{Acknowledgments}

Funding: This work was supported by National Natural Science Foundation of China $(81873673,81900841)$ the Fundamental Research Funds of the State Key Laboratory of Ophthalmology (30306020240020212), and the Young Teachers Training Program of Sun Yat-sen University (20ykpy143).

\section{Footnote}

Reporting Checklist: The authors have completed the TRIPOD reporting checklist. Available at http://dx.doi. org/10.21037/atm-20-1069

Data Sharing Statement: Available at http://dx.doi. org/10.21037/atm-20-1069

Conflicts of Interest: All authors have completed the ICMJE uniform disclosure form (available at http://dx.doi. org/10.21037/atm-20-1069). The authors have no conflicts of interest to declare.

Ethical Statement: The authors are accountable for all aspects of the work in ensuring that questions related to the accuracy or integrity of any part of the work are appropriately investigated and resolved. The study was conducted in accordance with the Declaration of Helsinki (as revised in 2013). The study was approved by the Institutional Board of Zhongshan Ophthalmic Centre of Sun Yat-sen University (IRB-ZOC-SYSU) and informed consent was taken from all the participants.

Open Access Statement: This is an Open Access article distributed in accordance with the Creative Commons Attribution-NonCommercial-NoDerivs 4.0 International License (CC BY-NC-ND 4.0), which permits the noncommercial replication and distribution of the article with the strict proviso that no changes or edits are made and the original work is properly cited (including links to both the formal publication through the relevant DOI and the license). See: https://creativecommons.org/licenses/by-nc-nd/4.0/.

\section{References}

1. Pyeritz RE. The Marfan syndrome. Annu Rev Med 2000;51:481-510.

2. Fuchs J. Marfan syndrome and other systemic disorders with congenital ectopia lentis. A Danish national survey. Acta Paediatr 1997;86:947-52.

3. Zeng Y, Li J, Li G, et al. Correction of the marfan syndrome pathogenic FBN1 mutation by base editing in human cells and heterozygous embryos. Mol Ther 2018;26:2631-7.

4. Sakai LY, Keene DR, Renard M, et al. FBN1: the diseasecausing gene for Marfan syndrome and other genetic disorders. Gene 2016;591:279-91.

5. Judge DP, Dietz HC. Marfan's syndrome. Lancet 2005;366:1965-76.

6. Faivre L, Collod-Beroud G, Loeys BL, et al. Effect of mutation type and location on clinical outcome in 1,013 probands with Marfan syndrome or related phenotypes and FBN1 mutations: an international study. Am J Hum Genet 2007;81:454-66.

7. Stheneur C, Tubach F, Jouneaux M, et al. Study of phenotype evolution during childhood in Marfan syndrome to improve clinical recognition. Genet Med 2014;16:246-50.

8. Habashi JP, Judge DP, Holm TM, et al. Losartan, an AT1 antagonist, prevents aortic aneurysm in a mouse model of Marfan syndrome. Science 2006;312:117-21.

9. Simon MA, Origlieri CA, Dinallo AM, et al. New management strategies for ectopia lentis. J Pediatr Ophthalmol Strabismus 2015;52:269-81.

10. Loeys BL, Dietz HC, Braverman AC, et al. The revised Ghent nosology for the Marfan syndrome. J Med Genet 2010;47:476-85.

11. Zhang Y, Wang Z, Huang S, et al. Parents' perceptions of diagnostic genetic testing for children with inherited retinal disease in China. Mol Genet Genomic Med 2019;7:e916.

12. Sridhar J, Chang JS. Marfan's syndrome with ectopia 
lentis. N Engl J Med 2017;377:1076.

13. Sandvik GF, Vanem TT, Rand-Hendriksen S, et al. Tenyear reinvestigation of ocular manifestations in Marfan syndrome. Clin Exp Ophthalmol 2019;47:212-8.

14. Beene LC, Traboulsi EI, Seven I, et al. Corneal deformation response and ocular geometry: a noninvasive diagnostic strategy in Marfan syndrome. Am J Ophthalmol 2016;161:56-64.e1.

15. Kinori M, Wehrli S, Kassem IS, et al. Biometry characteristics in adults and children with Marfan syndrome: from the Marfan Eye Consortium of Chicago. Am J Ophthalmol 2017;177:144-9.

16. Gehle P, Goergen B, Pilger D, et al. Biometric and structural ocular manifestations of Marfan syndrome. PLoS One 2017;12:e0183370.

17. Zhang Y, Jin G, Cao Q, et al. Distribution of axial length in Chinese congenital ectopia lentis patients: a retrospective study. BMC Ophthalmol 2017;17:113.

18. Drolsum L, Rand-Hendriksen S, Paus B, et al. Ocular findings in 87 adults with Ghent-1 verified Marfan syndrome. Acta Ophthalmol 2015;93:46-53.

19. Maumenee IH. The eye in the Marfan syndrome. Trans Am Ophthalmol Soc 1981;79:684-733.

20. Konradsen TR, Charlotta ZM. A descriptive study of ocular characteristics in Marfan syndrome. Acta Ophthalmol 2013;91:751-5.

21. Konradsen TR, Koivula A, Kugelberg M, et al. Corneal

Cite this article as: Wang Y, Lian Z, Zhou Y, Li X, Wu J, Zhang X, Jin G, Zheng D. Differential diagnosis of Marfan syndrome based on ocular biologic parameters. Ann Transl Med 2020;8(21):1354. doi: 10.21037/atm-20-1069 curvature, pachymetry, and endothelial cell density in Marfan syndrome. Acta Ophthalmol 2012;90:375-9.

22. Zheng D, Wan P, Liang J, et al. Comparison of clinical outcomes between iris-fixated anterior chamber intraocular lenses and scleral-fixated posterior chamber intraocular lenses in Marfan syndrome with lens subluxation. Clin Exp Ophthalmol 2012;40:268-74.

23. Luebke J, Boehringer D, Eberwein P, et al. Corneal $\mathrm{K}$-values as a diagnostic screening tool for Marfan syndrome. Cornea 2017;36:700-3.

24. Kara N, Bozkurt E, Baz O, et al. Corneal biomechanical properties and intraocular pressure measurement in Marfan patients. J Cataract Refract Surg 2012;38:309-14.

25. Ladewig MS, Robinson PN, Neumann LM, et al. Ocular manifestations and surgical results in patients with Marfan syndrome. Ophthalmologe 2006;103:777-82.

26. Sultan G, Baudouin C, Auzerie O, et al. Cornea in Marfan disease: Orbscan and in vivo confocal microscopy analysis. Invest Ophthalmol Vis Sci 2002;43:1757-64.

27. Heur M, Costin B, Crowe S, et al. The value of keratometry and central corneal thickness measurements in the clinical diagnosis of Marfan syndrome. Am J Ophthalmol 2008;145:997-1001.

28. Chen J, Jing Q, Tang Y, et al. Corneal curvature, astigmatism, and aberrations in Marfan syndrome with lens subluxation: evaluation by Pentacam HR System. Sci Rep 2018;8:4079. 